\title{
Paris law parameter identification based on the Extended Kalman Filter
}

\author{
M. Melgar ${ }^{1}$, C. Gomez-Jimenez ${ }^{2}$, L.D. Cot $^{1, a}$, S. Dejean ${ }^{3}$, C. Mabru ${ }^{1}$, and J. Martinez-Vega ${ }^{4}$ \\ 1 Institut Clment Ader ; Universit de Toulouse ; INSA ; 3, rue Caroline Aigle, 31400 Toulouse, France \\ 2 INSA ; Universit de Toulouse ; 135, avenue de rangueil, 31077 Toulouse, France \\ 3 Institut de Mathmatiques de Toulouse ; Universit de Toulouse ; UPS ; 118, route de Narbonne, 31062 Toulouse, France \\ 4 LAPLACE INP ; Universit de Toulouse ; UPS ; 118, route de Narbonne, 31062 Toulouse, France
}

\begin{abstract}
Aircraft structures are commonly subjected to repeated loading cycles leading to fatigue damage. Fatigue data can be extrapolated by fatigue models which are adopted to describe the fatigue damage behaviour. Such models depend on their parameters for accurate prediction of the fatigue life. Therefore, several methods have been developed for estimating the model parameters for both linear and nonlinear systems. It is useful for a broad class of parameter identification problems when the dynamic model is not known. In this paper, the Paris law is used as fatigue-crack-length growth model on a metallic component under loading cycles. The Extended Kalman Filter (EKF) is proposed as estimation method. Simulated crack length data is used to validate the estimation method. Based on experimental data obtained from fatigue experiment, the crack length and model parameters are estimated. Accurate model parameters allow a more realistic prediction of the fatigue life, consequently, the remaining useful life (RUL) of component can be accurately computed. In this sense, maintenance performance could be improved.
\end{abstract}

\section{Introduction}

Fatigue damage is one of the most probable modes of failure in aeronautical structures. During ground-air-ground cycles, in-service aircraft undergoes repeated pressurization and depressurization leading to fatigue loading cycles. Prediction of fatigue damage can prevent catastrophic failures. Currently, structural maintenance consists in timebased inspections integrated to overall aircraft timetable. The issue to improve operational reliability and reduce maintenance costs led to more cost-effective condition-based inspection approaches. Many investigations on structural health monitoring have shown that SHM is an appropriate tool for condition-based maintenance. On-board sensors and actuators assess structure health in real-time and allow to detect structural damages. Our purpose addresses accurate fatigue damage prognostic based on state-parameter estimation and experimental data in a metal specimen. The appropriate fatigue model for metal is the nonlinear Paris law depending upon uncertain parameters that describes the crack growth as a function of loading cycles [1] [2]. Usually, parameters are unknown because they are not measurable. Parameters associated to material properties are determined from experimental data. Parameter uncertainty can be observed if an experiment is repeated several times.

Many contributions based on parameter estimation have been presented. Among them, we can mention Coppe et al. [3] who have determined one of the two Paris law parameter using Bayesian inference and least-squares method with simulated noisy measurements; then, the RUL is computed. $\mathrm{Wu}$ and $\mathrm{Ni}[4]$ evaluated a stochastic fatigue damage parametric model whose parameters are deduced from experimental data and linear regression. Khelil et al.[5] have determined Paris law parameters using a linear regression

\footnotetext{
a e-mail: lea.cot@insa-toulouse.fr
}

method and experimental data from two aluminum alloy specimens. On the other hand, measurement instruments are tainted by random white noise which is combined to measurements and thus, affects the parameter estimation process. Such situation has led researchers to develop filtering methods such as the extended Kalman filter (EKF) for nonlinear systems [6][7][8]. The EKF progressively reduces the white noise in measurements and estimates both system state and associated parameters. Szabat et al. [7] have developed an algorithm to estimate various mechanical parameters of a mechanical control system. Lei et al.[8] proposed an estimating algorithm for detecting structural damage with limited measurements for civil structures. Singleton et al.[9] used the EKF to learn model parameters and to predict the RUL of bearing faults. In-service structures are subjected to fatigue loading under real-time and in-situ variable conditions. Probabilistic-statistical methods have been introduced to estimate parameters from experimental measurements [10]. Estimated parameters must reach true parameter values through real measurements. Combining physics-based damage models and available realtime measurements, parameter estimation might be performed. The estimation of both state and parameters is based on a joint system state-parameter vector also called the joint extended Kalman filter (JEKF) [11]. Corigliano et al. [12][13] showed that the JEKF provides the state variable and parameters estimation of a damage model for a structural system subject to dynamic loadings. Thus, the JEKF can be used to estimate both crack length and Paris law unknown parameters. Contributions of EKF estimation methods using fatigue crack models based on Paris law can be seen in [14] [15]. Based on accurate estimated parameters, the Paris law is able to provide a high-quality fatigue crack growth prediction. Knowing the behaviour of the crack growth, enables to predict the time to failure and 
therefore, the remaining useful life (RUL) of the component [10][16][3]. The identification of accurate parameters allows an accurate RUL prediction. As a result, the structural maintenance might be improved. In this paper, the JEKF is used to estimate both state and Paris law parameters using experimental data provided by fatigue loading experiment on steel specimen. The JEKF provides accurate parameter values. A sensitivity analysis is performed by first studying influence of process and measurement noises. The impact of initial estimation on parameters and initial variances of estimation error on parameters have been also studied. It is shown that the proposed modelling under initial conditions lead to accurate estimation results consistent with experimental data. The paper is organized as follows. In section 2, a single edge crack propagation experiment of a steel sample and measurement collection is explained. Section 3 details the JEKF estimation problem modelling. In section 4, results obtained are discussed followed by conclusions.

\section{Fatigue damage experiment}

Crack size real data have been obtained from a crack propagation experiment of a X38CrMoV5 steel sample under fatigue loading [17]. A single edge crack propagation experiment is considered. All specimens were machined and polished to obtain the final thickness $(0.6 \mathrm{~mm})$. More details of the specimen geometry and the experimental procedure can be consulted in [17]. Fatigue measurements obtained from this experiment (Fig. 1) have been used in the estimation process. Fatigue model is nonlinear dynamical

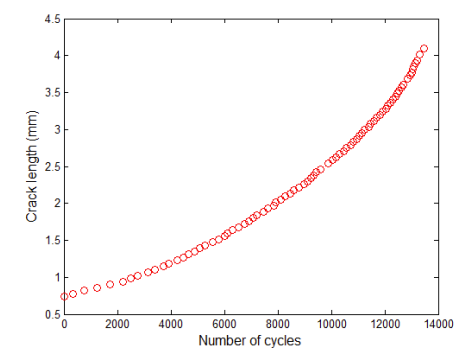

Fig. 1. Experimental fatigue crack length growth.

systems depending on model parameters. In the case of homogenous materials such as metal, the fatigue damage increases with applied cycles in a cumulative way. Currently, the Paris law is considered as fatigue crack growth model based on an ordinary differential equation expressing the crack size growth $l$ with the number of loading cycles $t$ applied to the structure

$$
\dot{l}(n)=C(\Delta K(l))^{m} .
$$

$C$ and $m$ are uncertain parameters also called Paris law parameters. Because of manufacturing process of structure, parameters $C$ and $m$ are variables and uncertainties arise. The stress intensity factor can be expressed as $\Delta K(l)=$ $g(l) l^{\frac{1}{2}}$ where function $g$ depends on the geometry of the specimen. The crack growth length is therefore expressed as

$$
\dot{l}(n)=C(g(l))^{m} l^{\frac{m}{2}} .
$$

In the case of the fatigue experiment explained in section 2 , function $g$ is defined as

$$
g(l)=\Delta \Sigma \sqrt{\pi} F\left(\frac{l}{w}\right)
$$

where $w$ is the specimen width, $\Delta \Sigma$ the loading applied and $F\left(\frac{l}{w}\right)$ the correction factor defined as

$$
\begin{aligned}
F\left(\frac{l}{w}\right)=1.0869+ & 0.2383 \frac{l}{w}+1.9830\left(\frac{l}{w}\right)^{2}-2.8373\left(\frac{l}{w}\right)^{3} \\
+ & 2.5771\left(\frac{l}{w}\right)^{4}
\end{aligned}
$$

In this case

$$
K(l)=\Delta \Sigma \sqrt{\pi} F\left(\frac{l}{w}\right) \sqrt{l} .
$$

\section{Estimation problem modelling}

\subsection{Joint extended Kalman filter in brief}

Let us set the general parametric model represented by the differentiable nonlinear function $f$ defined on $\mathbb{R}^{p} \times \mathbb{R}^{q}$ and values in $\mathbb{R}^{p}$ as

$$
\left\{\begin{array}{l}
\dot{y}=f(y, \Lambda) \\
y_{(0)}=y_{0}
\end{array}\right.
$$

where $\Lambda=\left(\lambda^{(1)}, \ldots, \lambda^{(q)}\right)$ is the unknown parameter vector.

\subsection{Extended Kalman Filter formulation}

Let us consider the discrete-time process governed by the nonlinear stochastic equations at time $k$

$$
\left\{\begin{array}{l}
x_{k}=f\left(x_{k-1}, \Lambda_{k-1}\right)+w_{k-1} \\
z_{k}=h\left(x_{k}\right)+v_{k}
\end{array}\right.
$$

where $f$ and $h$ are nonlinear differentiable functions, $x \in$ $\mathbb{R}^{p}$ the state variable, $\Lambda \in \mathbb{R}^{q}$ the unknown parameter vector and $z \in \mathbb{R}^{p}$ the measurement vector. $w$ and $v$ are process and measurement noise assumed to be additive and zero mean Gaussian white. The estimation of both state and parameters requires the use of the joint extended Kalman filter by constructing the augmented state vector $X \in \mathbb{R}^{p+q}$ such that $X_{k}=\left(x_{k} \Lambda_{k}\right)^{T}$ at time $k$. Assuming that parameters are constant during the process, the JEKF is now based on equations

$$
\left\{\begin{array}{l}
x_{k}=f\left(x_{k-1}\right)+w_{k-1} \\
\Lambda_{k}=i d\left(\Lambda_{k-1}\right) \\
z_{k}=h\left(X_{k}\right)+v_{k}
\end{array}\right.
$$

The JEKF requires the computation of the Jacobian matrix $J_{k}\left(\hat{X}_{k-1}\right)$ at time $k$ relative to the augmented state $X_{k}$ evaluated at the last estimate $\hat{X}_{k-1}=\left(\hat{x}_{k-1}, \hat{\Lambda}_{k-1}\right)^{T}$, i.e. at time $k-1$. Because $J_{i d, k}\left(\hat{x}_{k-1}\right)=0_{q, n}$ and $J_{i d, k}\left(\hat{\Lambda}_{k-1}\right)=I_{q}$, the Jacobian matrix is expressed as

$$
J_{k}\left(\hat{X}_{k-1}\right)=\left(\begin{array}{cc}
J_{f, k}\left(\hat{x}_{k-1}\right) & J_{f, k}\left(\hat{\Lambda}_{k-1}\right) \\
0_{q, n} & I_{q}
\end{array}\right)
$$

where $J_{f, k}\left(\hat{x}_{k-1}\right)=\left.\frac{\partial f^{(i)}(x, \Lambda)}{\partial x^{(j)}}\right|_{\left(x=\hat{x}_{k-1}, \Lambda=\hat{\Lambda}_{k-1}\right)}, \forall 1 \leq i, j \leq n$, and $J_{f, k}\left(\hat{\Lambda}_{k-1}\right)=\left.\frac{\partial f^{(i)}(x, \Lambda)}{\partial \lambda^{(j)}}\right|_{\left(x=\hat{x}_{k-1}, \Lambda=\hat{\Lambda}_{k-1}\right)}, \forall 1 \leq i \leq q, \quad 1 \leq j \leq n$. Only the crack length increase is observed. So, in 7 , the third equation become

$$
z_{k}=H X_{k}+v_{k}
$$

where the sensitivity matrix $H \in M_{p, p+q}(\mathbb{R})$ is $H=\left(\begin{array}{lll}1 & 0 & 0\end{array}\right)^{T}$. The EKF standard algorithm is then applied to solve system (7). 


\subsection{Application to the Paris law}

In this case, the joint vector is $X=(l, C, m)^{T}$ and the vector to be estimated is $\Lambda=(C, m)^{T}$. The first recurrent equation in (7) is obtained by approximating the rate of damage growth in Eq.1 by a finite difference. We therefore obtain $l\left(n_{k}\right)=f\left(l\left(n_{k-1}\right), \Lambda_{k-1}\right)$ where function $f$ is defined as

$$
\begin{aligned}
f\left(l\left(n_{k-1}\right), \Lambda_{k-1}\right) & \approx l\left(n_{k-1}\right)+C g\left(\left(n_{k-1}\right)\right)^{m} l\left(n_{k-1}\right)^{\frac{m}{2}}\left(n_{k}-n_{k-1}\right), \\
f\left(l_{k-1}, \Lambda_{k-1}\right) & \approx l_{k-1}+C g\left(l_{k-1}\right)^{m} l_{k-1}^{\frac{m}{2}}\left(n_{k}-n_{k-1}\right),
\end{aligned}
$$

by setting $l\left(n_{k}\right)=l_{k}, \quad \forall k \in \mathbb{N}$. The Jacobian matrix is expressed as

$$
J_{k}\left(\hat{X}_{k-1}\right)=\left(\begin{array}{ccc}
J_{f, k}\left(\hat{l}_{k-1}\right) & J_{f, k}\left(\hat{C}_{k-1}\right) & J_{f, k}\left(\hat{m}_{k-1}\right) \\
0 & 1 & 0 \\
0 & 0 & 1
\end{array}\right)
$$

$J_{f, k}\left(\hat{l}_{k-1}\right)=\left.\frac{\partial f(l, \Lambda)}{\partial l}\right|_{\left(l=\hat{l}_{k-1}, \Lambda=\hat{\Lambda}_{k-1}\right)}, J_{f, k}\left(\hat{C}_{k-1}\right)=\left.\frac{\partial f(l, \Lambda)}{\partial C}\right|_{\left(l=\hat{l}_{k-1}, \Lambda=\hat{\Lambda}_{k-1}\right)}$ and $J_{f, k}\left(\hat{m}_{k-1}\right)=\left.\frac{\partial f(l, \Lambda)}{\partial m}\right|_{\left(l=\hat{l}_{k-1}, \Lambda=\hat{\Lambda}_{k-1}\right)}$.

As shown in section (4.1), this modelling do not lead to satisfying results due to exponent of parameter $m$ in Eq. 9. Another modelling is therefore addressed by considering the joint vector $X=(\log l, \log C, m)^{T}$ and the vector of parameters to be estimated $\Lambda=(\log C, m)^{T}$. In this case, the new state function is defined as

$$
\begin{aligned}
& f\left(\log l_{k-1}, \Lambda_{k-1}\right) \approx \log l_{k-1}+ \exp (\log C+m \log g(l) \\
&\left.+\left(\frac{m}{2}-1\right) \log l_{k-1}\right) \Delta n_{k}
\end{aligned}
$$

where $g(l)=\Delta \Sigma \sqrt{\pi} F\left(\frac{l}{w}\right)$ and $\Delta n_{k}=n_{k}-n_{k-1}$.

By taken into account expressions (3) of function $F$ and its first derivative $\dot{F}$, and setting $\beta=\Delta \Sigma \sqrt{\pi}$, the Jacobian partial derivatives are expressed as follow

$$
\begin{gathered}
J_{f, k}\left(\hat{l}_{k-1}\right)=1+\left[\hat{m}_{k-1} \frac{\dot{F}\left(\frac{\hat{l}_{k-1}}{w}\right)}{F\left(\frac{\hat{l}_{k-1}}{w}\right)}+\frac{\hat{m}_{k-1}}{2}-1\right] \\
. \exp \left(\hat{C}_{k-1}+\hat{m}_{k-1} \log \left(\beta F\left(\frac{\hat{l}_{k-1}}{w}\right)\right)+\left(\frac{\hat{m}_{k-1}}{2}-1\right) \hat{l}_{k-1}\right) \Delta n_{k}, \\
J_{f, k}\left(\hat{C}_{k-1}\right)=\exp \left(\hat{C}_{k-1}+\hat{m}_{k-1} \log \left(\beta F\left(\frac{\hat{l}_{k-1}}{w}\right)\right)+\left(\frac{\hat{m}_{k-1}}{2}-1\right) \hat{l}_{k-1}\right) \Delta n_{k}, \\
J_{f, k}\left(\hat{m}_{k-1}\right)=\left[\log \left(\beta F\left(\frac{\hat{l}_{k-1}}{w}\right)\right)+\frac{1}{2} \hat{l}_{k-1}\right] \\
\exp \left(\hat{C}_{k-1}+\hat{m}_{k-1} \log \left(\beta F\left(\frac{\hat{l}_{k-1}}{w}\right)\right)+\left(\frac{\hat{m}_{k-1}}{2}-1\right) \hat{l}_{k-1}\right) \Delta n_{k} .
\end{gathered}
$$

Based on equations (7), state function of Eq. 11 and Jacobian coefficients of Eq. 12, the EKF algorithm is applied to estimate the joint vector $X$ that includes the Paris parameters. Results are provided in section 4.2.

\section{Results and discussion}

\subsection{Results}

Using real data allows us to recreate a real situation where measurements can be obtained by an SHM system. Our main interest is identified the better results in terms of the estimation error on real measurements. The estimations of crack length and parameters are carry out using around 78 real measurements of crack length. In general terms, the crack length estimation can presents a slight convergence to measurement over time, it is taking into account that there is a few number of measurements below 4000 cycles. However, parameter estimations don't converge to expected values satisfactorily. The JEKF algorithm was initiated with the process noise covariance matrix $Q=$ $10^{-3} * I_{3}$, measurement noise variance $R=10^{14}$ and the diagonal elements of the estimation error variance matrix $P_{0}=\{1,2.5 E 3,2 E 4\}$. High values for $R$ and $P_{0}$ were considered with the propose to reach an accuracy on the estimation of the parameters $C$ and $m$. However, the estimation error on $C$ is unstable without reaching a notable trend. The estimation error on $m$ increases with very small variations without achieving satisfactory results. In term of the computational charge, the estimation process takes more time by finding the appropriate initial conditions. Furthermore, the estimators of both parameters don't converge to expected values. We can say that both the initial conditions and nonlinearity of the system affect the estimation process. It is known that the logarithms are one of the most important mathematical tools for the linearisation of exponential functions. In this paper, we propose another modelling based on the logarithmic transformation of the state function, see Eq.11, to improve the accuracy of the parameter estimations.

\subsection{Logarithmic transformation based new results}

Here results are presented using JEKF algorithm based on logarithmic transformation. The JEKF algorithm is adjusted to start up with initial conditions considered, around 50 tests were developed. The estimations can be unstable and difficult convergence to expected values $\left(C=2 \times 10^{-10}\right.$ and $m=1.63$ ). However, the estimators of the parameters can achieve satisfactory results. The simulations were organised by increasing the initial conditions for the parameters $C$ and $m$, and the initial estimation error variance of both parameters. Values of $Q$ and $R$ are fixed to $Q / R=0.01$ ratio to learn and to analyse the impact of the initial conditions of the parameters in the estimation process. We show that the precision on both parameter estimation errors depends on the process initialization which also impact the cycle for which a required precision is reached. The estimation process was observed by the last estimations, estimation error variances and absolute errors of both parameters. In Fig. 2-a depicts the last estimations made of parameter $C$ when the initial estimation error variance on the parameter $C\left(P_{0_{22}}\right)$ is increased. When $P_{0_{22}}$ grows, the estimation of $C$ moves away from to expected value over time. For $P_{0_{22}}<1$ approximately, the estimator of $C$ is closed to expected value. In another way, if estimation error variance on parameter $m\left(P_{0_{33}}\right)$ is increased, the estimator of $m$ converge to expected value over time, see Fig. 2-b. That is to say, the estimator of $m$ closes to expected value when the $P_{0_{33}}$ increases over time. We observe that the initial variances for both parameters can affect the speed of the estimation process. In other words, $P_{0_{22}}$ and $P_{0_{33}}$ can influence that the JEKF algorithm achieves large or small values of the last estimation error variance. For instances, Fig. 3-a 


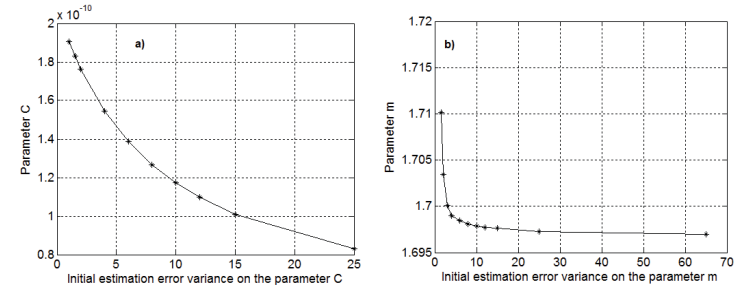

Fig. 2. a) Last estimation of parameter $C$ and b) Last estimation of parameter $m$.

depicts that estimation error variance on $C$ reaches large values when the $P_{0_{22}}$ increases. In contrast with the last estimation error variance on $m$, see Fig. 3-b, this reaches small values with low variations. Therefore, the variation of the initial estimation error variance on $C$ considerably can affects the parameter estimation process with increasing the last estimation error variances on $C$ and $m$. It means that appropriate initialisation of variance on the parameter $C$ can has more influence in the estimation process due to that it is a coefficient of the model. In this paper, we have
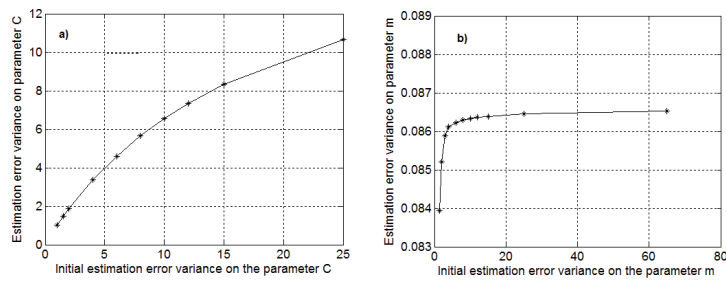

Fig. 3. a) Estimation error variance on $C$ and b) Estimation error variance on $m$.

used the finite difference approximations for modelling the crack length evolution. Fig. 4 depicts the crack length evolution by exact solution and finite difference of Paris law model. There is that evolution trends are closed over time. Therefore, estimation of $l$ should converges to the expected trend. In Fig. 5-a shows a summary of the estimation errors on $l$. We can observed that the estimation error of T3, T4 and T5 quickly converge to zero and has closed values. It means that they have the same $Q / R$ ratio. The trend of estimation error on $l$ can be minimized for small values of $R$. After 4000 cycles, the estimation error on $l$ convergences and after 10000 cycles, it is better. However, estimations of the parameters $C$ and $m$ present $\mathrm{o}=$ another trends to expected values but in some instant they approximate to best values. In Fig. 5-b, we can observed that best estimations of parameter $C$ are found for T6 and T7 with values $Q=0.0001 * I_{3}$ and $R=0.1$ before to 4000 cycles, and $Q=0.0001 * I_{3}$ and $R=1$ before 7000 cycles, respectively. Another trends show not a decreasing until zero, the estimation errors remain above 2 . On the other hand, the best estimations of parameter $m$ are found for T6 before 4000 cycles and T2, T4, T5 and T7 after 7000 cycles. Therefore, we can select the estimation of T6 and T7 because both the estimation errors of parameter $C$ and $m$ converge to zero before at 4000 cycles and between 6500 and 7500 cycles, respectively. However, both estimation errors of parameter $C$ and $m$ by the T7, the trends show that the estimators are more stable than the estimators by test T6 which increase quickly. Estimation error on $C$ remains stable and relatively small after 6000 cycles compared with the other tests. If we stop the updating of the estimation of $m$ when its estimation error reaches the value more near to zero, best estimation is identified between 6500 and 7500 cycles.

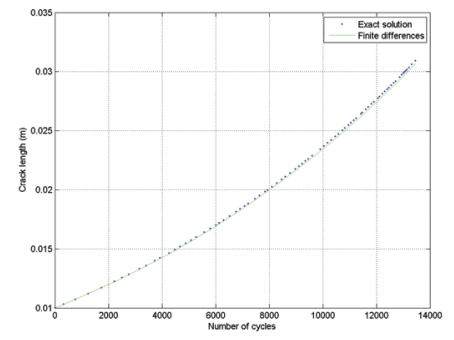

Fig. 4. Crack length evolution by exact solution and finitedifference method.
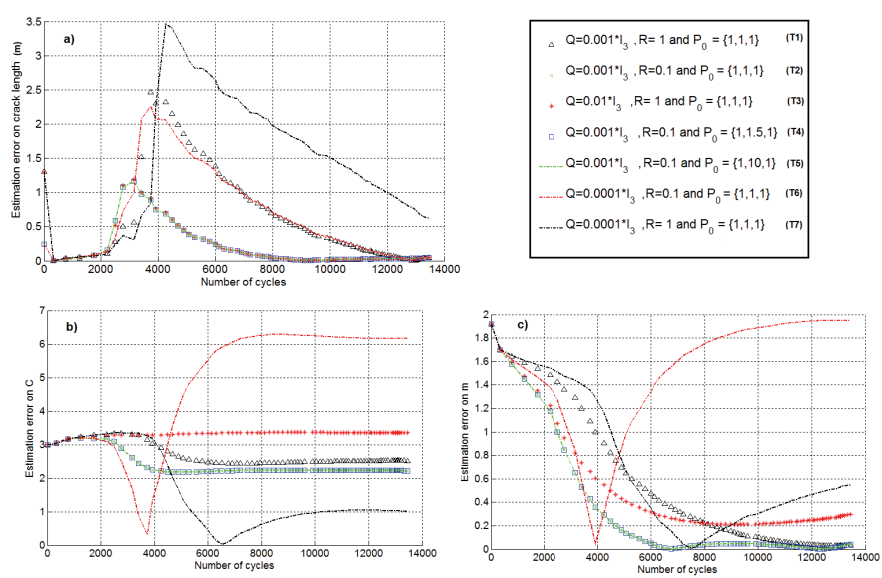

Fig. 5. a) Summary of estimation error on $l$. b) Summary of estimation errors on $C$. c) Summary of estimation errors on $m$.

\section{Conclusion}

In this paper real data was used to estimate Paris law parameters. Finite-difference method used to discretised the Paris law model and approximating the crack length evolution. An adaptive estimation algorithm was applied to the problem of fatigue crack propagation, in order to identify the best model parameters for improving of the prediction of the crack length. Using real data, results have been presented and discussed. The Extended Kalman Filter (EKF) by the joint formulation and logarithmic transformation was developed with promising estimations. Paris law parameters were estimated in order to analyse the impact of the initial conditions in the estimation process. The results show that estimators eventually converge to the expected values in specific instants. Here pretty accuracies can be obtained by appropriate initialisation of the parameters $C$ and $m$ and their estimation error variances. The initial estimation error variance plays an important roll to estimate the parameters with small variation in the estimation error variances. Results show that the estimators of $C$ and $m$ can be lightly confidence at a specific instant. Important contributions have been made for the parameter identification problems. Considering real data allows simulating real situations where the important measurements given by an SHM system are used to estimate unknown model parameters for predicting the future condition of a monitored structural component and estimating its RUL. 


\section{References}

1. SM Beden, S Abdullah, and AK Ariffin. European Journal of Scientific Research, 28:364-397, 2009.

2. JC Newman, EP Phillips, and MH Swain. International Journal of fatigue, 21:109-119, 1999.

3. A Coppe, MJ Pais, RT Haftka, and NH Kim. Journal of Aircraft, 49:1965-1973, 2012.

4. WF $\mathrm{Wu}$ and $\mathrm{CC}$ Ni. Probabilistic Engineering Mechanics, 18:107-118, 2003.

5. F Khelil, B Aour, M Belhouari, and N Benseddiq. Engineering, Technology $\mathcal{E}$ Applied Science Research, 3:pp-488, 2013.

6. J Hu, Z Wang, H Gao, and LK Stergioulas. Automatica, 48:2007-2015, 2012.

7. K Szabat and T Orlowska-Kowalska. Industrial Electronics, IEEE Transactions on, 55:1075-1084, 2008.

8. Y Lei, Y Jiang, and Z Xu. Mechanical Systems and Signal Processing, 28:229-243, 2012.

9. RK Singleton, EG Strangas, and S Aviyente. Industrial Electronics, IEEE Transactions on, 62:17811790, 2015.

10. E Zio and F Di Maio. Expert Systems with Applications, 39:10681-10692, 2012.

11. LD Cot and R Lozi. International Journal of Chaotic Computing, 2:3-13, 2013.

12. A Corigliano and S Mariani. Computer Methods in Applied Mechanics and Engineering, 193:3807-3835, 2004.

13. S Mariani and A Corigliano. Computer methods in applied mechanics and engineering, 194:5242-5272, 2005.

14. A Ray and S Tangirala. Control Systems Technology, IEEE Transactions on, 4:443-451, 1996.

15. AC Cobb, JE Michaels, TE Michaels, Donald O Thompson, and Dale E Chimenti. In Aip Conference Proceedings, volume 975, page 1252, 2008.

16. A Coppe, RT Haftka, and NH Kim. In 12th AIAA NonDeterministic Approaches Conference, pages 12-15, 2010.

17. M Shah, C Mabru, and F Rezaï-Aria. Fatigue $\mathcal{E}$ Fracture of Engineering Materials $\mathcal{E}$ Structures, 38:742754, 2015. 